\title{
¿Puede usarse el receptor soluble de transferrina para diagnosticar anemia ferropénica y evaluar la respuesta al hierro en lactantes con desnutrición aguda moderada?

\author{
Can soluble transferrin receptor be used in diagnosing iron \\ deficiency anemia and assessing iron response in infants with
} moderate acute malnutrition?
}

\author{
Dra. Bahar Büyükkaragöza, Dr. Necat A. Akgun ${ }^{b}$ Dra. Ayse D. Bulusc, \\ Dra. Sultan Durmus Aydogdud y Dr. Cengiz Bale
}

a. Departamento de Pediatría, Eskişehir Osmangazi Üniversitesi, Eskişehir, Turquía

b. Departamento de Hematología Pediátrica, Eskişehir Osmangazi Üniversitesi, Eskişehir, Turquía

c. Departamento de Endocrinología Pediátrica, Hospital Universitario y de Investigación de Keçiören, Ankara, Turquía

d. Departamento de Metabolismo y Nutrición en Pediatría, Eskişehir Osmangazi

Üniversitesi, Eskişehir, Turquía

e. Departamento de Bioestadística, Eskişehir Osmangazi

Üniversitesi, Eskişehir, Turquía.

Correspondencia:

Dra. Bahar Büyükkaragöz:

karamanbahar@yahoo.com

Financiamiento:

Ninguno.

Conflicto de intereses: Ninguno que declarar.

Recibido: 4-6-2016

Aceptado: 13-10-2016

Cómo citar: Büyükkaragöz B, Akgun NA, Bulus AD, et al. ¿Puede usarse el receptor soluble de transferrina para diagnosticar anemia ferropénica y evaluar la respuesta al hierro en lactantes con desnutrición aguda moderada? Arch Argent Pediatr 2017;115(2):125-132.

\section{RESUMEN}

Objetivo. Evaluar la eficacia del receptor soluble de transferrina (RST)enel diagnóstico dela anemia ferropénica $(\mathrm{AF})$ y en la evaluación dela respuesta al hierro en los lactantes con desnutrición aguda moderada (DAM).

Población y métodos. Se reclutó a lactantes con valores dehemoglobina $(\mathrm{Hb})$ inferioresalos valores umbrales de anemia para su edad y con anemia hipocrómica/microcítica observada en el frotis de sangre periférica. La DAM se definió como un puntaje $Z$ de peso/estatura de entre $<-2$ y -3 . Se compararon los valores del hemograma, los parámetros férricos y el RST entre 41 lactantes con DAMy anemia (grupoDA),32lactantesconanemia $\sin$ DAM (grupo A) y controles saludables $(n=30)$. Una vez completado el tratamiento de la anemia y la desnutrición, se repitieron las evaluaciones. Resultados. Además delosíndices hematológicos compatibles con $\mathrm{AF}$, los valores de hierro sérico (Fe) y saturación de transferrina (ST) eran significativamente menores, mientras queel valor detransferrina era significativamentemayoren los grupos DA y A en comparación con los controles $(p<0,001)$. Los valores de ferritina y proteína C-reactiva (PCR) eran significativamente más elevados en el grupo DA $(p<0,05$ para la ferritina, $p<0,01$ para la PCR). El valor medio del RST fue similar en ambos grupos (DA y A) $(p>0,05)$ y significativamente mayor que en los controles ( $p$ $<0,001)$. Después del tratamiento con hierro, el RST disminuyó en los grupos DA y A $(p<0,001)$ a valores similares a los observados en los controles. El RST se correlacionó negativamente con la $\mathrm{Hb}$ durante todo el estudio (grupo DA: $r=-0,350, p$ $<0,05$; grupo A: $\mathrm{r}=-0,683, p<0,01$ ).

Conclusiones. Dado que los valores del RST en los grupos DA y A disminuyeron después del tratamiento con hierro, consideramos que este parámetro no estuvo afectado por la DAM ni la inflamación y puede usarse, por sí solo, para detectar la AF y supervisar la respuesta al tratamiento en los lactantes con DAM.

Palabras clave: receptor de transferrina, anemia ferropénica, desnutrición, lactantes.

http://dx.doi.org/10.5546/aap.2017.125

Texto completo en inglés:

http:/ /dx.doi.org/10.5546/aap.2017.eng.125

\section{INTRODUCCIÓN}

La desnutrición por carencias energéticas y proteínicas se manifiesta principalmente por una ingesta inadecuada de proteínas y calorías. ${ }^{1}$ Es un problema de salud muy importante en los países en vías de desarrollo, y está asociada con el $50 \%$ al $60 \%$ de la mortalidad en menores de 5 años en estos entornos. ${ }^{2}$ Además de causar retraso del crecimiento, emaciación y peso insuficiente, las deficiencias de micronutrientes, en especial de vitaminas y minerales, también son componentes importantes de la desnutrición por carencias energéticas y proteínicas. ${ }^{13,4}$ La ferropenia es la carencia nutricional más frecuente en todo el mundo; además, se observan anemia por inflamación y AF en los niños con desnutrición. . $^{4-6}$

En la actualidad, las pruebas tradicionales para el diagnóstico de la AF no bastan. ${ }^{7} \mathrm{Si}$ se usan por separado, ninguna es lo suficientemente sensible o específica como para diagnosticar la AF. Por un lado, la sensibilidad del volumen corpuscular medio (VCM) es baja; por el otro, la ST y la capacidad total de fijación del hierro (CTFH) tienen una baja especificidad. ${ }^{8}$ Además, estas pruebas también pueden estar afectadas por ciertas afecciones que las tornan inadecuadas para el diagnóstico de la AF. El Fe, la CTFH, la ferritina y la transferrina son proteínas de la fase aguda que pueden estar elevadas en presencia de procesos infecciosos o inflamatorios concurrentes. ${ }^{8-12}$ 
Cada vez más estudios investigan la utilidad del RST para diferenciar la anemia en niños. ${ }^{13-16}$ El RST es una forma extracelular truncada del receptor de transferrina transmembranario (una glucoproteína que se expresa principalmente en las células que requieren hierro) producido mediante proteólisis. ${ }^{17} \mathrm{La}$ cantidad de receptores tisulares, en su mayoría células eritroides, determina la cantidad de RST. ${ }^{18}$ La concentración plasmática de RST refleja la densidad del receptor en las células y la cantidad de células que expresan el receptor; también está estrechamente relacionada con las demandas celulares de hierro y la tasa de proliferación de células eritroides. ${ }^{17} \mathrm{El}$ valor de RST aumenta a medida que disminuyen los niveles celulares de hierro, ${ }^{15}$ no se ve afectado por la inflamación y es adecuado para supervisar la respuesta eritropoyética al tratamiento de la $\mathrm{AF}$ con hierro. ${ }^{18-21}$

Hasta el momento, no se han realizado estudios que se centren en el uso del RST en el diagnóstico diferencial de la AF en niños con desnutrición. Nuestro objetivo era evaluar la eficacia del RST para detectar la AF y examinar la respuesta al tratamiento con hierro en los lactantes con DAM, además de determinar si es superior a los marcadores tradicionales de la AF en estos pacientes.

\section{POBLACIÓN Y MÉTODOS}

Se inscribió en este estudio prospectivo a 73 lactantes con anemia de entre 6 y 24 meses de edad, que fueron atendidos en el Departamento de Pediatría de la Eskişehir Osmangazi Üniversitesi durante un año. Se seleccionó a 30 lactantes en el mismo grupo etario (20 niños y 10 niñas) sin anemia ni desnutrición como controles en el grupo de referencia (grupo $\mathrm{C}$ ). Un valor de $\mathrm{Hb}$ por debajo del valor umbral de anemia para la edad específica se consideró anemia. ${ }^{22}$ Se reclutó solamente a los pacientes con anemia hipocrómica/microcítica observada en, al menos, dos frotis de sangre periférica evaluados por operadores diferentes. Se midieron los valores del hemograma, Fe, transferrina, ST, ferritina y RST para realizar un diagnóstico adecuado de AF y excluir otras causas de anemia hipocrómica/ microcítica. Los valores de VCM $<70$ fl, ST $<15 \%$, Fe $<36 \mu \mathrm{g} / \mathrm{dl}$ y ferritina $<10-12 \mathrm{ng} / \mathrm{ml}$ se consideraron valores de corte para el diagnóstico de AF. ${ }^{6,22}$ También se midieron los valores de vitamina B12, ácido fólico y reticulocitos.

Se registraron las mediciones antropométricas (peso, estatura, perímetros cefálico y mesobraquial) de los participantes incluidos en los grupos de estudio. Se subdividió al grupo de pacientes según la presencia de desnutrición. Se consideró que los lactantes con un puntaje $Z$ de peso/estatura de entre $<-2$ y -3 tenían DAM. ${ }^{1,23}$ Se incluyó a 41 pacientes (18 niños y 23 niñas) con anemia y DAM (grupo DA) y a 32 pacientes (27 niños y 5 niñas) con anemia sin DAM (grupo A).

Se excluyó a los pacientes con las siguientes afecciones: deshidratación o problemas para alimentarse por vía oral, antecedentes de hemorragia aguda o transfusiones de sangre o ingesta de hierro o factores eritropoyéticos en los tres meses previos, desnutrición grave (puntaje $Z$ de peso/estatura $<-3$ ), síndrome de absorción insuficiente debidamente definido, otro tipos de anemia hipocrómica/microcítica (talasemias $\alpha$ y $\beta$, anemia sideroblástica, anemia causada por afecciones inflamatorias prolongadas), anemia megaloblástica debido a carencia de vitamina B12 y ácido fólico. Se explicó el objetivo de este estudio claramente a los padres antes de obtener el consentimiento. El estudio fue aprobado por el comité de ética local correspondiente al hospital.

Se registraron los factores de riesgo para el desarrollo de AF correspondientes al período prenatal (presencia de anemia o hemorragia materna, ingesta de preparaciones a base de hierro), el nacimiento (edad gestacional y peso al nacer) y el período posnatal (antecedentes de lactancia, contenido de los alimentos en el período de destete y uso previo de suplementos con hierro).

A los grupos DA y A se les administró tratamiento con preparaciones de $6 \mathrm{mg} / \mathrm{kg}$ de hierro elemental (sulfato ferroso). Además, al grupo DA también se le administraron preparaciones proteínicas y energéticas, y suplementos con multivitaminas, magnesio, zinc y ácido fólico. A los pacientes se les realizaron chequeos mensuales durante tres meses. Dado que 7 pacientes en el grupo DA y 6 en el grupo A interrumpieron su participación durante el control, al final del período de seguimiento se repitieron las mediciones antropométricas y los análisis de sangre en 34 y 26 pacientes de los grupos DA y A, respectivamente.

\section{Obtención de muestras de sangre y análisis de laboratorio}

Se obtuvieron muestras de sangre periférica por venopunción estándar mediante técnica aséptica. El hemograma se realizó en un analizador automático Coulter Gen-S con kits Beckman-Coulter. Los valores de vitamina B12, ácido fólico, Fe y ferritina se evaluaron en un analizador automático Hitachi 
Roche. La transferrina se analizó en el analizador automático IMAGE (Beckman-Coulter). La ST (\%) se calculó de la siguiente manera: Fe/CTFH x 100; donde la CTFH se estima como $25,1 \times$ transferrina. Los sueros de las muestras de sangre obtenidos para el análisis de RST se congelaron y almacenaron en tubos Eppendorf de $2 \mathrm{ml} \mathrm{a}$ $-20{ }^{\circ} \mathrm{C}$ hasta el momento del análisis. El RST se analizó mediante enzimoinmunoanálisis de tipo sándwich en Diagnostic Grifols SA (Valles, España) con un analizador automático de ELISA con kits ELISA de RST humano (BioVendor Laboratory Medicine, Inc.).

\section{Análisis estadístico}

Las variables continuas se describieron como media $\pm \mathrm{DE}$. Se usó la prueba de KolmogorovSmirnov para evaluar la normalidad de las variables. Para el análisis estadístico, se usaron la prueba U de Mann-Whitney y la prueba $t$ en muestras independientes para comparar ambos grupos. La prueba del orden con signo de Wilcoxon y la prueba $t$ en muestras emparejadas se usaron para comparar las muestras relacionadas. Las variables categóricas se resumieron como recuentos (porcentajes) y se compararon con la prueba de $\chi^{2}$ y la prueba exacta de Fisher. Se usó el análisis de correlación de Pearson para determinar la correlación entre los parámetros. Los datos se analizaron con el paquete estadístico SPSS, versión 15.0 (SPSS Inc. Chicago, IL). Un valor de $p<0,05$ se consideró estadísticamente significativo.

\section{RESULTADOS \\ Mediciones antropométricas}

Según lo previsto, al inicio del estudio, la media del peso, el puntaje $\mathrm{Z}$ de peso/estatura y los perímetros cefálico y mesobraquial del grupo DA eran significativamente más bajos que en los grupos A y C $(p<0,001)$. Después del período de seguimiento de tres meses, si bien se observó un aumento significativo en todos los índices antropométricos del grupo DA $(p<0,05$ puntaje $\mathrm{Z}$ de peso/estatura), los valores eran aún significativamente menores que en los grupos $\mathrm{A}$

TABLA 1. Mediciones antropométricas al inicio y al final del estudio

\begin{tabular}{|c|c|c|c|c|c|c|c|c|c|c|}
\hline & $\begin{array}{l}\text { DA (1) } \\
(n=41)\end{array}$ & $\begin{array}{l}\text { DA (2) } \\
(\mathrm{n}=34)\end{array}$ & $\mathrm{p} 1$ & $\begin{array}{c}A(1) \\
(n=32)\end{array}$ & $\begin{array}{c}A(2) \\
(n=26)\end{array}$ & p2 & $\begin{array}{c}\text { Controles (1) } \\
(n=30)\end{array}$ & $\begin{array}{c}\text { Controles (2) } \\
(\mathbf{n}=25)\end{array}$ & p3 & $\mathrm{p} 4$ \\
\hline $\begin{array}{l}\text { Sexo (n) } \\
\text { (niñas/niños) }\end{array}$ & $23 / 18$ & $20 / 14$ & & $5 / 27$ & $3 / 23$ & & $10 / 20$ & $7 / 18$ & & \\
\hline Edad (meses) & $11,8 \pm 4,1$ & $14,9 \pm 2,7$ & $<0,001$ & $13,1 \pm 5,0$ & $16,0 \pm 1,7$ & $<0,001$ & $13,3 \pm 5,3$ & $16,2 \pm 3,7$ & $<0,001$ & 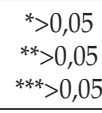 \\
\hline Peso (g) & $\begin{array}{c}7476,1 \pm 139 \\
4,7\end{array}$ & $\begin{array}{c}8738,2 \pm 113 \\
3,7\end{array}$ & $<0,001$ & $\begin{array}{c}10203,7 \pm 16 \\
41,3\end{array}$ & $\begin{array}{c}11294,2 \pm 16 \\
29,9\end{array}$ & $<0,001$ & $\begin{array}{c}10667,7 \pm 18 \\
06,4\end{array}$ & $\begin{array}{c}11501,2 \pm 13 \\
31,8\end{array}$ & $<0,001$ & $\begin{array}{l}*<0,001 \\
* *<0,001 \\
* * *>0,05\end{array}$ \\
\hline Estatura (cm) & $72,2 \pm 6,1$ & $74,9 \pm 4,4$ & $<0,001$ & $75,9 \pm 6,8$ & $80,1 \pm 5,4$ & $<0,001$ & $74,5 \pm 6,6$ & $80,4 \pm 6,1$ & $<0,001$ & $\begin{array}{l}*<0,05 \\
* *<0,05 \\
* * *>0,05\end{array}$ \\
\hline $\mathrm{PC}(\mathrm{cm})$ & $43,7 \pm 2,4$ & $44,9 \pm 1,8$ & $<0,001$ & $46,4 \pm 2,6$ & $47,6 \pm 2,2$ & $<0,001$ & $46,3 \pm 1,7$ & $47,4 \pm 3,5$ & $<0,001$ & $\begin{array}{l}*<0,001 \\
* *<0,001 \\
* * * * 0,05\end{array}$ \\
\hline PMB (cm) & $12,4 \pm 1,3$ & $13,5 \pm 1,4$ & $<0,001$ & $14,9 \pm 1,3$ & $15,8 \pm 1,1$ & $<0,001$ & $14,0 \pm 1,8$ & $15,1 \pm 1,4$ & $<0,001$ & 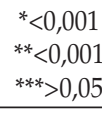 \\
\hline $\begin{array}{l}\text { Puntaje Z de } \\
\text { peso/estatura }\end{array}$ & $-2,3 \pm 1,4$ & $-1,5 \pm 1,6$ & $<0,05$ & $0,2 \pm 0,2$ & $0,3 \pm 0,4$ & $>0,05$ & $0,8 \pm 0,3$ & $0,6 \pm 0,2$ & $>0,05$ & $\begin{array}{l}*<0,001 \\
* *<0,001 \\
* * *>0,05\end{array}$ \\
\hline
\end{tabular}

PC: perímetro cefálico; PMB: perímetro mesobraquial

DA (1): grupo de desnutrición al inicio; DA (2): grupo de desnutrición al final;

A (1): grupo de anemia al inicio; A (2): grupo de anemia al final

p1: diferencia entre DA (1) y DA (2)

p2: diferencia entre A (1) y A (2)

p3: diferencia entre Controles (1) y Controles (2)

$\mathrm{p} 4^{*}$ : diferencia entre DA (1) y A (1); p $4^{* *}$ : diferencia entre DA (1) y Controles (1); p4*** diferencia entre A (1) y Controles (1) 
y $C^{*}$. En este período, el $14,7 \%$ de los pacientes en el grupo DA $(n=5)$ aún tenían DAM. Por otro lado, los puntajes $Z$ de peso/estatura de los grupos A y $C$ seguían siendo similares al final del seguimiento* (Tabla 1). A pesar de la pequeña cantidad de pacientes que aún tenían DAM al final del seguimiento, el aumento significativo de los puntajes $Z$ de peso/estatura del grupo DA se atribuye principalmente al tratamiento eficaz de la desnutrición más que al crecimiento regular.

\section{Factores de riesgo para el desarrollo de AF en el período prenatal, el nacimiento $y$ el período posnatal}

Se observaron antecedentes de prematurez en
8 lactantes del grupo de pacientes (5 casos en el grupo DA y 3 en el grupo A) y en 2 de los controles. Además, se observó bajo peso al nacer (BPN) en 14 lactantes del grupo de pacientes (10 casos en el grupo DA y 4 en el grupo A) y en 3 controles (Tabla 2). A pesar de los valores más bajos, no se evaluó por separado a los lactantes prematuros y con BPN de toda la población de estudio ya que las mediciones antropométricas no diferían estadísticamente respecto de los lactantes nacidos a término ni de los que tenían un peso normal al nacer*.

La cantidad de casos de anemia materna en el período prenatal era significativamente mayor en el grupo de pacientes que entre los controles $(p<0,001)$. Si bien el porcentaje de

* No se muestran los datos.

TABLA 2. Factores de riesgo para el desarrollo de AF

\begin{tabular}{lccc}
\hline & $\begin{array}{c}\text { Pacientes } \\
(\mathbf{n = 7 3 )} \mathbf{( \% )}\end{array}$ & $\begin{array}{c}\text { Controles } \\
(\mathbf{n = 3 0 )} \mathbf{( \% )}\end{array}$ & $p$ \\
\hline Hemorragia materna (n) & $10(13,7)$ & $1(3,3)$ & $>0,05$ \\
Anemia materna (n) & $44(60,3)$ & $11(36,7)$ & $<0,001$ \\
Tratamiento prenatal con hierro (n) & $42(57,5)$ & $13(43,3)$ & $>0,05$ \\
BPN (n) & $14(19,2)$ & $3(10,0)$ & $>0,05$ \\
Prematurez (n) & $8(11,1)$ & $2(6,7)$ & $>0,05$ \\
Lactancia materna solamente durante & & & $>0,05$ \\
los primeros 6 meses (n) & $22(27,4)$ & $8(26,7)$ & $>0,05$ \\
Lactancia materna y destete temprano (n) & $47(64,4)$ & $22(73,3)$ & $<0,001$ \\
Uso de leche de vaca & $45(61,6)$ & $8(26,7)$ & $<0,05$ \\
Alimentos que contienen harina de arroz (n) & $36(49,3)$ & $6(20,0)$ & $<0,05$ \\
Uso de productos cárnicos (n) & $14(19,2)$ & $14(46,7)$ & $>0,05$ \\
Uso previo de suplementos con hierro (n) & $18(24,6)$ & $13(43,3)$ & \\
\hline
\end{tabular}

BPN: bajo peso al nacer

TABLA 3. Valores hematológicos al inicio y al final del estudio

\begin{tabular}{|c|c|c|c|c|c|c|c|c|c|}
\hline & $\begin{array}{l}\text { DA (1) } \\
(\mathrm{n}=41)\end{array}$ & $\begin{array}{l}\text { DA (2) } \\
(\mathrm{n}=34)\end{array}$ & p1 & $\begin{array}{c}\text { A (1) } \\
(n=32)\end{array}$ & $\begin{array}{c}A(2) \\
(n=26)\end{array}$ & $\mathrm{p} 2$ & $\begin{array}{c}\text { Controles } \\
(n=30)\end{array}$ & p3 & $\mathrm{p} 4$ \\
\hline $\mathrm{Hb}(\mathrm{g} / \mathrm{dl})$ & $9,6 \pm 0,9$ & $11,5 \pm 1,2$ & $<0,001$ & $9,4 \pm 0,9$ & $11,9 \pm 0,8$ & $<0,001$ & $12,0 \pm 0,6$ & $\begin{array}{l}*<0,001 \\
* *<0,001 \\
\end{array}$ & $\begin{array}{l}*>0,05 \\
* *>0,05\end{array}$ \\
\hline HCT (\%) & $29,4 \pm 2,8$ & $34,0 \pm 3,4$ & $<0,001$ & $29,0 \pm 2,4$ & $34,9 \pm 2,4$ & $<0,001$ & $31,0 \pm 3,7$ & $\begin{array}{l}*<0,001 \\
* *<0,001\end{array}$ & $\begin{array}{l}*>0,05 \\
* *>0,05\end{array}$ \\
\hline VCM (fl) & $69,1 \pm 7,0$ & $73,2 \pm 5,9$ & $<0,001$ & $63,5 \pm 7,4$ & $72,9 \pm 4,6$ & $<0,001$ & $75,7 \pm 4,4$ & $\begin{array}{l}*<0,001 \\
* *<0,001\end{array}$ & $\begin{array}{l}{ }^{*}>0,05 \\
* *>0,05\end{array}$ \\
\hline HCM (pg) & $22,9 \pm 2,8$ & $24,8 \pm 2,4$ & $<0,001$ & $20,8 \pm 3,1$ & $24,9 \pm 1,8$ & $<0,001$ & $26,1 \pm 1,8$ & $\begin{array}{l}*<0,001 \\
* *<0,01\end{array}$ & $\begin{array}{l}*>0,05 \\
* *>0,05\end{array}$ \\
\hline ADE (\%) & $17,0 \pm 3,3$ & $16,6 \pm 3,2$ & $>0,05$ & $18,2 \pm 3,6$ & $16,3 \pm 3,7$ & $<0,05$ & $14,3 \pm 2,0$ & $\begin{array}{l}*<0,001 \\
* * 0,001\end{array}$ & $\begin{array}{l}*<0,001 \\
* *<0,001\end{array}$ \\
\hline
\end{tabular}

DA (1): grupo de desnutrición al inicio; DA (2): grupo de desnutrición al final;

A (1): grupo de anemia al inicio; A (2): grupo de anemia al final

p1: diferencia entre DA (1) y DA (2)

p2: diferencia entre A (1) y A (2)

$\mathrm{p} 3^{*}$ : diferencia entre DA (1) y Controles; $\mathrm{p} 3^{* *}$ : diferencia entre A (1) y Controles

$\mathrm{p}^{*}$ : diferencia entre DA (2) y Controles; $4^{* *}$ : diferencia entre A (2) y Controles

$\mathrm{Hb}$ : hemoglobina; HCT: hematocrito; VCM: volumen corpuscular medio; HCM: hemoglobina corpuscular medio;

ADE: ampliltud de distribución eritrocitaria. 
lactancia en los primeros 6 meses de vida era muy alto en este estudio, también fue frecuente el destete temprano. Los nutrientes utilizados para el destete variaron. Los alimentos de transición no fortificados con hierro (en especial, los alimentos preparados con harina de arroz) y la leche de vaca no maternizada fueron mucho más frecuentes en el grupo de pacientes; en cambio, los productos cárnicos después de los 6 meses de vida fueron significativamente más comunes entre los controles $(p<0,05$, Tabla 2$)$.

Al momento del ingreso, se observaron infecciones concurrentes (infección respiratoria, gastroenteritis aguda, infección urinaria, etc.) en el $78,0 \%$, el $71,8 \%$ y el $50,0 \%$ de los participantes del grupo DA, A y C, respectivamente. Se observó cardiopatía congénita en el $24,4 \%(n=10)$ de los pacientes del grupo DA. No se identificaron enfermedades crónicas concurrentes en los grupos A y C.

\section{Análisis hematológico del grupo de estudio}

Al inicio del estudio, los valores de $\mathrm{Hb}$, hematocrito (HCT), VCM, hemoglobina corpuscular media (HCM), Fe y ST eran significativamente más bajos en los grupos DA y A que en los controles $(p<0,001)$. Entretanto, los valores de transferrina y amplitud de la distribución eritrocitaria (ADE) eran significativamente mayores en los grupos DA y A que en los controles $(p<0,001)$. A pesar de ser similar en el grupo A y en los controles, la ferritina era significativamente más alta en el grupo que en el grupo A y en los controles ( $p$ $<0,05)^{*}$. Durante este período, el valor medio del RST era similar en los grupos DA y A $(p>0,05)^{*} \mathrm{y}$ significativamente mayor que en los controles $(p<$ $0,001)$. Los valores hematológicos y los parámetros férricos se muestran en las Tablas 3 y 4 .

Los valores de los análisis de laboratorio, incluidas las pruebas funcionales hepáticas y renales, los valores de electrólitos en sangre, proteínas séricas, albúmina, vitamina B12, ácido fólico y reticulocitos eran similares $(p>0,05)$ y normales en todos los grupos. En cambio, la proteína C-reactiva (PCR) era elevada en los grupos DA $(1,8 \pm 3,1 \mathrm{mg} / \mathrm{dl})$ y A $(0,8 \pm 1,3 \mathrm{mg} /$ d1) y normal en los controles $(0,5 \pm 1,7)$ (intervalo normal: 0-0,5 mg/dl). Además, era significativamente más elevada en el grupo DA que en los demás grupos $(p<0,01)$.

Al final del tratamiento para la desnutrición y la AF durante tres meses, los valores de $\mathrm{Hb}$, HCT y ST aumentaron significativamente, y el valor del RST disminuyó de manera significativa en los grupos DA y A $(p<0,001)$. Excepto por el valor persistentemente elevado de ADE, todos los parámetros hematológicos de los grupos DA y A eran similares a los de los controles en este período. Mientras tanto, la ferritina había disminuido en el grupo DA $(p>0,05)$ y aumentado en el grupo A $(p<0,05)$ hacia el final del período de seguimiento (Tablas 3 y 4 ).

Si bien no se observó una correlación sólida, se

TABla 4. Parámetros férricos al inicio y al final del estudio

\begin{tabular}{|c|c|c|c|c|c|c|c|c|c|}
\hline & $\begin{array}{l}\text { DA (1) } \\
(\mathrm{n}=41)\end{array}$ & $\begin{array}{l}\text { DA (2) } \\
(\mathrm{n}=34)\end{array}$ & $\mathrm{p} 1$ & $\begin{array}{c}\mathrm{A}(1) \\
(\mathrm{n}=32)\end{array}$ & $\begin{array}{c}A(2) \\
(n=26)\end{array}$ & p2 & $\begin{array}{c}\text { Controles } \\
(\mathrm{n}=30)\end{array}$ & p3 & $\mathrm{p} 4$ \\
\hline Ferritina $(\mathrm{ng} / \mathrm{ml})$ & $62,1 \pm 124,1$ & $44,7 \pm 109,8$ & $>0,05$ & $23,0 \pm 21,6$ & $33,2 \pm 18,5$ & $<0,05$ & $33,9 \pm 17,3$ & $\begin{array}{l}*<0,05 \\
* *>0,05\end{array}$ & $\begin{array}{l}*>0,05 \\
* *>0,05 \\
\end{array}$ \\
\hline RST ( $\mathrm{gg} / \mathrm{ml})$ & $2,7 \pm 1,6$ & $1,6 \pm 0,8$ & $<0,001$ & $3,0 \pm 1,1$ & $1,4 \pm 0,4$ & $<0,001$ & $1,6 \pm 0,5$ & $\begin{array}{c}*<0,001 \\
* *<0,001\end{array}$ & $\begin{array}{l}{ }^{*}>0,05 \\
* *>0,05\end{array}$ \\
\hline $\mathrm{Fe}(\mu \mathrm{g} / \mathrm{dl})$ & $29,9 \pm 13,3$ & $60,9 \pm 45,0$ & $<0,001$ & $29,1 \pm 14,5$ & $59,9 \pm 43,2$ & $<0,001$ & $51,37 \pm 29,8$ & $\begin{array}{l}*<0,001 \\
* *<0,001\end{array}$ & $\begin{array}{l}*>0,05 \\
* *>0,05\end{array}$ \\
\hline $\begin{array}{l}\text { Transferrina } \\
\text { (mg/dl) }\end{array}$ & $282,3 \pm 67,1$ & $245,9 \pm 65,8$ & $<0,01$ & $311,8 \pm 58,3$ & $245,4 \pm 54,4$ & $<0,001$ & $244,5 \pm 46,3$ & $\begin{array}{l}*<0,001 \\
* *<0,001\end{array}$ & $\begin{array}{l}*>0,05 \\
* *>0,05\end{array}$ \\
\hline ST (\%) & $9,5 \pm 4,7$ & $21,0 \pm 16,4$ & $<0,001$ & $7,9 \pm 4,3$ & $19,9 \pm 15,5$ & $<0,001$ & $20,1 \pm 18,5$ & $\begin{array}{l}{ }^{*}<0,001 \\
* *<0,001\end{array}$ & $\begin{array}{l}*>0,05 \\
* *>0,05\end{array}$ \\
\hline
\end{tabular}

DA (1): grupo de desnutrición al inicio; DA (2): grupo de desnutrición al final;

A (1): grupo de anemia al inicio; A (2): grupo de anemia al final

p1: diferencia entre DA (1) y DA (2)

p2: diferencia entre A (1) y A (2)

p3*: diferencia entre DA (1) y Controles; $3^{* *}$ : diferencia entre A (1) y Controles

$\mathrm{p} 4^{*}$ : diferencia entre DA (2) y Controles; $4^{* *}$ : diferencia entre A (2) y Controles

RST: receptor soluble de transferrina

ST: saturación de transferrina. 
notó que el RST se correlacionaba negativamente con la $\mathrm{Hb}$ en los grupos DA y A (grupo DA: $r=$ $-0,350, p<0,05$; grupo A: $\mathrm{r}=-0,683, p<0,01)$.

\section{DISCUSIÓN}

Hasta la fecha, no existe una única prueba de laboratorio para reconocer la ferropenia. ${ }^{13}$ En la actualidad, además de un valor bajo de $\mathrm{Hb}$, VCM y HCM, se aceptan la disminución de la ST y la ferritina como herramientas útiles importantes para el diagnóstico de la AF. ${ }^{24}$ Sin embargo, las pruebas tradicionales usadas para detectar la ferropenia siguen siendo insuficientes y, excepto la ferritina, su fiabilidad diagnóstica es baja. En cuanto al costo general, también existen informes que favorecen su uso. ${ }^{8}$

Hasta donde sabemos, no se han realizado estudios que evalúen la validez de los parámetros férricos tradicionales para diagnosticar la AF en los niños con desnutrición. Además, algunos parámetros como la transferrina o la ferritina podrían verse alterados por hipoproteinemia o inflamación concurrente en los niños con desnutrición por carencias energéticas y proteínicas. ${ }^{11,25}$ Por lo tanto, el uso de marcadores más confiables y fáciles de constatar constituiría una mejor estrategia para detectar la AF en los pacientes con desnutrición.

En este estudio, los parámetros tradicionales usados como marcadores de la AF, por ejemplo, $\mathrm{Hb}, \mathrm{HCT}, \mathrm{Fe}$ sérico y ST, eran significativamente más bajos en los grupos DA y A que en los controles al inicio del estudio. Sin embargo, todos pueden estar disminuidos en el caso de anemia por inflamación. En este período, los valores de PCR eran altos en ambos grupos. Por otro lado, después de la administración de suplementos con hierro durante tres meses, todos los parámetros férricos alcanzaron valores similares a los de los controles. Con base en estos hallazgos, se concluye que los pacientes de los grupos DA y A tenían anemia combinada ( $\mathrm{AF}+$ anemia por inflamación) al inicio del estudio.

El grupo de pacientes eran lactantes con anemia hipocrómica/microcítica. Si bien la anemia por inflamación es, en su mayoría, de tipo normocrómica/normocítica, se sabe que la anemia hipocrómica/microcítica también puede presentarse con afecciones inflamatorias prolongadas. ${ }^{26}$ En este estudio, no se reclutó a pacientes con afecciones inflamatorias crónicas (por ejemplo, enfermedades renales, reumatológicas o gastrointestinales). En el grupo DA, 10 pacientes tenían cardiopatía acianótica congénita subyacente, pero todos estaban siendo debidamente controlados, y ninguno tenía insuficiencia cardíaca congestiva que requiriera medicamentos. Por lo tanto, creemos que no se incluyó en nuestro estudio a pacientes con anemia hipocrómica/microcítica causada por afecciones inflamatorias prolongadas.

Si bien no se usa habitualmente en el diagnóstico de la AF debido a su costo elevado, la concentración elevada de transferrina es una manifestación frecuente de la AF. Mientras tanto, puede ser baja en los pacientes con desnutrición por hipoproteinemia. ${ }^{10,25}$ En nuestro estudio, a pesar de estar dentro de los límites normales, los valores de transferrina eran significativamente más altos en el grupo DA que en los controles $(p<0,001)$. No se observó hipoproteinemia en el grupo DA (proteínas totales: 6,6 $\pm 0,73 \mathrm{~g} / \mathrm{dl}$ ), $\mathrm{y}$ fue posible identificar la AF con base en los valores de transferrina.

Una concentración baja de ferritina es el hallazgo inicial de la AF. Sin embargo, puede llegar a una concentración de $30 \mathrm{ng} / \mathrm{ml} \mathrm{a}$ $50 \mathrm{ng} / \mathrm{ml}$ en la AF asociada con enfermedades infecciosas o inflamatorias. ${ }^{27}$ En este estudio, la ferritina estaba significativamente más elevada en el grupo DA que en los grupos A y C. Dado que la PCR también era más alta en el grupo DA que en el resto de los grupos, se interpretó el aumento de la ferritina en el grupo DA como resultado de infecciones concurrentes. Por lo tanto, la ferritina no fue útil para el diagnóstico de la AF en el grupo DA. Asimismo, a pesar de la administración de suplementos con hierro, la concentración de ferritina disminuyó en el grupo DA hacia el final del seguimiento. Esta observación se atribuye principalmente a la mejoría clínica en lugar de a la ineficacia del tratamiento con hierro.

El receptor soluble de transferrina es útil para evaluar la deficiencia de hierro tisular y las afecciones con estimulación de la eritropoyesis, como la anemia hemolítica, la esferocitosis hereditaria, la anemia drepanocítica, la anemia megaloblástica o la eritropoyesis ineficaz. ${ }^{15,19,28-32}$ Al inicio del estudio, todos los pacientes tenían anemia hipocrómica/microcítica y valores normales de ácido fólico, vitamina B12 y reticulocitos. El valor del RST era bastante similar en los grupos DA y A, aunque significativamente más elevado que en los controles. Además, se correlacionaba negativamente con los valores de $\mathrm{Hb}$. Por lo tanto, consideramos que el RST por sí solo es eficaz para demostrar la presencia de AF en el grupo de pacientes. 
En un estudio realizado en adultos, se demostró que la desnutrición no afectaba la concentración de RST, independientemente del estado del hierro. ${ }^{33}$ En nuestro estudio, el grupo de pacientes (grupos DA y A) tenía valores de RST muy cercanos, sin tener en cuenta la presencia de desnutrición. Sin embargo, dado que no se inscribió un grupo de pacientes con DAM e hipoproteinemia, simplemente podemos concluir que el RST no se vio afectado en los lactantes con desnutrición y concentraciones normales de proteínas.

En informes previos, se indicó que el RST disminuye en respuesta al tratamiento con hierro; por lo tanto, puede usarse para supervisar la respuesta eritropoyética a la administración de suplementos con hierro. ${ }^{19,34}$ Del mismo modo, al final del tratamiento con hierro durante tres meses, el RST disminuyó en los grupos DA y A, y alcanzó valores similares a los de los controles. En consecuencia, consideramos que también es útil para demostrar la eficacia del tratamiento con hierro, independientemente de la desnutrición no resuelta en algunos lactantes.

Se informó que el receptor sérico de transferrina no se ve afectado por infecciones agudas, inflamaciones o enfermedades crónicas concurrentes. ${ }^{19}$ En este estudio, si bien la PCR era significativamente más alta en el grupo DA que en el resto, el RST estaba aumentado de forma similar en el grupo A. Este hallazgo respalda aún más el hecho de que el RST no se vio afectado por la presencia de afecciones inflamatorias agudas.

Los niños con AF pueden tener elevación de la ADE incluso cuatro semanas después de iniciar la administración de suplementos con hierro. ${ }^{35}$ En nuestro estudio, todos los índices hematológicos de los grupos DA y A alcanzaron valores similares a los de los controles al final de los tres meses de tratamiento con hierro, excepto el valor persistentemente elevado de ADE. Dado que no es posible explicar decididamente el motivo de los valores elevados de ADE al final de estudio, consideramos que es posible que se necesite un período más prolongado que el informado previamente para lograr la normalización de la ADE.

Nuestro estudio presenta ciertas limitaciones. No se incluyó un grupo puro de AF (con valores de ferritina francamente disminuidos y sin inflamación) ni un grupo de pacientes con DAM e hipoproteinemia. Además, debido al rango etario limitado de los grupos de estudio, no es posible extrapolar los resultados a toda la población pediátrica. Creemos que estudios donde se incluya a todos estos grupos de pacientes revelarían mejores resultados.

\section{CONCLUSIÓN}

En este estudio, el valor del RST no se vio afectado por la presencia de desnutrición e inflamación concurrente. Asimismo, una vez corregida la AF, el valor del RST disminuyó a niveles similares a los de los controles. Como resultado, consideramos que puede usarse el RST como marcador confiable de la AF en los lactantes con DAM. En lugar de usar los parámetros de AF tradicionales, recomendamos medir solamente el RST para diagnosticar la AF y evaluar la eficacia del tratamiento con hierro en los niños con DAM. Se requieren estudios futuros con una muestra más grande para confirmar estos resultados.

\section{REFERENCIAS}

1. Alderman $H$, Shekar M. Nutrition, food security and health security. En: Kliegman RM, Stanton BF,ST. Geme JW, Schor NF, Behrman RE, eds. Nelson Textbook of Pediatrics. 19th ed. Philadelphia: Elsevier Saunders; 2011.Págs.170-9.

2. Ubesie A, Ibeziakor N. High burden of protein-energy malnutrition in Nigeria: beyond the health care setting. Ann Med Health Sci Res 2012;2(1):66-9.

3. Bhutta ZA. Micronutrient needs of malnourished children. Curr Opin Clin Nutr Metab Care 2008;11(3):309-14.

4. Grover Z, Ee LC. Protein energy malnutrition. Pediatr Clin North Am 2009;56(5):1055-68.

5. Fondu P, Hariga-Muller C, Mozes N, Neve J, et al. Proteinenergy malnutrition and anemia in Kivu. Am J Clin Nutr 1978;31(1):46-56.

6. ÖzkaleM,Sipahi T.Hematologic and bonemarrow changes in children with protein-energy malnutrition. Pediatr Hematol Oncol 2014;31(4):349-58.

7. Lopez A, Cacoub P, Macdougall IC, Peyrin-Biroulet L. Iron deficiency anaemia. Lancet 2016;387(10021):907-16.

8. Burns ER, Goldberg SN, Lawrence C, Wenz B. Clinical utility of serum tests for iron deficiency in hospitalized patients. Am J Clin Pathol 1990;93(2):240-5.

9. Özdemir N. Iron deficiency anemia from diagnosis to treatment in children. Turk Pediatri Ars 2015;50(1):11-9.

10. SazawalS, Dhingra U,Dhingra P,Dutta A, et al. Efficiency of red cell distribution width in identification of children aged 1-3 years with iron deficiency anemia against traditional hematological markers. BMC Pediatr 2014;14:8.

11. Radcliffe LE, Thomas W, Glen J, Padhi S, et al. Diagnosis and Management of Iron Deficiency in CKD: A Summary of the NICEGuideline Recommendations and Their Rationale. Am J Kidney Dis 2016;67(4):548-58.

12. Thurnham DI, McCabe LD, Haldar S, Wieringa FT, et al. Adjusting plasma ferritin concentrations to remove the effects of subclinical inflammation in the assessment of iron deficiency: a meta-analysis. Am J Clin Nutr 2010;92(3):546-55.

13. Kamer B, Dółka E, Pasowska R, wi tkowska E. The usefulness of soluble transferrin receptor (sTfR) in differentiating anemia occurring in young children. Folia Histochem Cytobiol 2012;50(3):473-9.

14. Ho CH. The diagnostic role of serum transferrin receptor in patients with various anemia. Zhonhua Yi Xue Za Zhi 
(Taipei) 2002;65(2):55-60.

15. Yoon SH, Kim DS, Yu ST, Shin SR, et al. The usefulness of soluble transferrin receptor in the diagnosis and treatment of iron deficiency anemia in children. Korean J Pediatr 2015;58(1):15-9.

16. DimitriouH,StiakakiE,MarkakiEA,BolonakiI,etal.Soluble Transferrin receptor levels and soluble transferrin receptor / $\log$ ferritin index in the evaluation of erythropoietic status in childhood infections and malignancy. Acta Paediatr 2000;89(10):1169-73.

17. Braga F, Infusino I, Dolci A, Panteghini M. Soluble transferrin receptor in complicated anemia. Clin Chim Acta 2014;431:143-7.

18. Harms K, KaiserT. Beyond soluble transferrin receptor: old challenges and new horizons. Best Pract Res Clin Endocrinol Metab 2015;29(5):799-810.

19. Beguin Y. Soluble transferrin receptor for the evaluation of erythropoiesis and iron status. Clin Chim Acta 2003;329(12):9-22.

20. Ferguson BJ, Skikne BS, Simpson KM, Baynes RD, et al. Serum transferrin receptor distinguishes the anemia of chronic disease from iron deficiency anemia. J Lab Clin Med 1992;119(4):385-90.

21. BhaskaramP,Madhavan NairK, Balakrishna N, RavinderP, etal.Serum transferrin receptor in children with respiratory infections. Eur J Clin Nutr 2003;57(1):75-80.

22. Comité Nacional de Hematología. Anemia ferropénica. Guía de diagnóstico y tratamiento. Arch Argent Pediatr 2009;107(4):353-61.

23. World Health Organization. Management of severe malnutrition: a manual for physicians and other senior health workers, WHO, Geneva 1999. [Acceso: 19 de noviembre de 2009]. Disponible en: http: / / apps.who.int/ iris/bitstream/10665/41999/1/a57361.pdf

24. Subramaniam G, Girish M. Iron deficiency anemia in children. Indian J Pediatr 2015;82(6):558-64.
25. Withold W, Neumayer C, Beyrau R, Heins M, et al. Efficacy of transferrin determination in human sera in the diagnosis of iron deficiency. Eur J Clin Chem Clin Biochem 1994; 32(1): 19-25.

26. Ganz T, Nemeth E. Iron sequestration and anemia of inflammation. Semin Hematol 2009;46(4):387-93.

27. Baillie FJ, Morrison AE, Fergus I. Soluble transferrin receptor: a discriminating assay for iron deficiency. Clin Lab Haematol 2003;25(6):353-7.

28. Flowers CH, Skikne BS, Covell AM, Cook JD. The clinical measurement of serum transferrin receptor. J Lan Clin Med 1989;114(4):368-77.

29. Choi JW, Pai SH. Associations between serum transferrin receptor concentrations and erythropoietic activities according to body iron status. Am Clin Lab Sci 2003;33(3): 279-84.

30. Skikne BS, Flowers CH, Cook JD. Serum transferrin receptor: a quantitative measure of tissue iron deficiency. Blood 1990;75(9):1870-6.

31. Ahluwalia N. Diagnostic utility of serum transferrin receptors measurement in assessing iron status. Nutr Rev 1998;56(5 Pt 1):133-41.

32. Olivares M,Walter T,CookJD, HertrampfE, etal.Usefulness of serum transferrin receptor and serum ferritin in diagnosis of iron deficiency in infancy. Am J Clin Nutr 2000;72(5): 1191-5.

33. Kuvibidila S, Warrier RP, Ode D, Yu L. Serum transferrin receptor concentrations in women with malnutrition. Am J Clin Nutr 1996;63(4):596-601.

34. Kohgo Y, Niitsu Y, Kondo H, Kato J. Serum transferrin receptor as a new index of erythropoiesis. Blood 1987;70(6):1955-8.

35. AslanD, GümrükF, Gürgey A, Altay C. Importance of RDW value in differential diagnosis of hypochrome anemias. $A m$ J Hematol 2002;69(1):31-3.

\section{Novedades}

Desde el anterior número de Febrero, Archivos Argentinos de Pediatría incorporó como suplementos a los Consensos y Guías que elaboran las Subcomisiones, Comités y Grupos de trabajo. Esto permitirá facilitar el acceso y además, que cada uno de los artículos dispongan los datos completos que permitirán que sean citados de acuerdo a los criterios internacionales. Asimismo, de esta forma, los consensos y guías van a estar incluidos en el Pubmed, un aspecto sumamente importante para expandir su difusión a nivel internacional. 